\title{
Publisher's Note: Strong coupling on a forbidden transition in strontium and nondestructive atom counting [Phys. Rev. A 93, 023804 (2016)]
}

Matthew A. Norcia and James K. Thompson

(Received 9 February 2016; published 19 February 2016)

DOI: 10.1103/PhysRevA.93.029902

This paper was published online on 2 February 2016 with an incorrect author footnote. Matthew A. Norcia's footnote should read as "matthew.norcia@colorado.edu." The author footnote has been corrected as of 5 February 2016. The author footnote is correct in the printed version of the journal. 